\title{
Lyme Carditis and Complete Heart Block: An Unusual Case of a Young Student
}

\author{
Sherrie Khadanga ${ }^{a, b}$, Samreen Raza ${ }^{a}$
}

\begin{abstract}
Lyme carditis is a relatively uncommon medical condition in young people. It can be accompanied by myalgias, sweating episodes, fever and chest pain for several weeks. It sometimes has certain manifestations masquerading as pericarditis. Varying degrees of atrioventricular (AV) block accompanied by rhythm abnormalities are not uncommon. We describe an unusual case of a young male college student presenting with shortness of breath and evidence of complete heart block secondary to the rare diagnosis of complete heart block due to Lyme disease causing cardiac conduction abnormalities. This condition completely resolved with intravenous antibiotic therapy. This case represents a relevant and unique clinical scenario which is an important education point highlighting prompt diagnosis in a reversible cause of complete heart block.
\end{abstract}

Keywords: Lyme disease; Complete heart block; Atrioventricular block

\section{Introduction}

Lyme disease is a vector-borne illness caused by spirochete Borrelia burgdorferi and is primarily transmitted by the Ixodes tick. It is most prevalent in the northeast and upper midwestern states in the United States [1]. This systemic disease is also common in central and eastern Europe and especially the Scandinavian countries [2,3]. While the number of confirmed cases in the United States from 2003 to 2012 was between 20,000 and 30,000, Lyme carditis occurs in only $1 \%$

Manuscript accepted for publication July 4, 2014

${ }^{a}$ Department of Medicine, University of Vermont, Burlington, VT, USA

${ }^{b}$ Corresponding Author: Sherrie Khadanga, Department of Medicine,

University of Vermont, 111 Colchester Avenue, Fletcher 311,

Burlington, VT 05401, USA. Email: sherrie.khadanga@vtmednet.org

doi: http://dx.doi.org/10.14740/jmc1841w of Lyme disease cases [4]. Cardiovascular manifestations often emerge within 3 weeks of exposure and most commonly result in second or third degree atrioventricular (AV) block that resolves spontaneously within days or weeks [5]. In this particularly young patient, a total cardiac block was found and promptly diagnosed. The patient's inability to recall any tick bite or rash made the situation intriguing.

\section{Case Report}

A healthy 19-year-old male presented to the emergency department after a syncopal event. Prior to this episode, he had been experiencing myalgias, drenching sweats, low-grade fever and chest pain for the past several weeks. One week before his emergency department visit, he began to develop dizziness, dyspnea on exertion and worsening fatigue. He denied any sick contacts or recent travel but upon questioning mentioned going camping 2 months ago. In the emergency department, he was noted to have orthostatic hypotension. Pertinent findings of his physical exam included bradycardia with no cardiac murmur. Abdominal exam revealed right upper quadrant tenderness. There were no neurological deficits or evidence of skin rash. Initial electrocardiogram demonstrated Mobitz type $1 \mathrm{AV}$ block and right bundle branch block. Bedside echocardiogram demonstrated a normal ejection fraction and mild aortic insufficiency. Three hours later, a serial electrocardiogram demonstrated progression to complete heart block with a heart rate between 30 and $40 \mathrm{~s}$ but remained hemodynamically stable. Liver function testing revealed a mild transaminitis with ALT of 79 and AST of 31 with a normal total bilirubin of 0.6. Although the patient denied recent tick bite and did not present with rash, Lyme titers were sent due to presence of new AV heart block and fever. Suspicious of Lyme pericarditis, he was started on $2 \mathrm{~g}$ of intravenous (IV) ceftriaxone daily and remained in third degree heart block with a prolonged PR interval. Following administration of antibiotics, the patient's heart block resolved back to normal sinus rhythm. Subsequently, laboratory testing returned positive for Lyme antibody (IgM positive; IgG positive with three bands). The patient made a complete recovery and was discharged on oral doxycycline for 21 days 
and experienced complete resolution of his symptoms.

\section{Discussion}

Lyme disease is a multi-systemic illness that is usually diagnosed clinically and confirmed by serological testing. Often times, the diagnosis is made based on a history of tick bite, presence of a distinct rash (erythema chronicum migrans) and acute systemic illness [6]. Previous studies indicated that $4-10 \%$ of untreated Lyme disease patients exhibit cardiac manifestations, the most frequent of which is a transient AV nodal block [7]. However, current CDC data indicate a lower rate of Lyme carditis of $1 \%$. This patient falls into this low incidence category. The infection can affect the conduction system around the AV node as well as the outer or inner membranes of the heart. In the majority of cases, the heart block is mild or incomplete leading to symptoms such as lightheadedness, chest pain and shortness of breath, all of which this patient experienced. Most cases resolve with appropriate antibiotic therapy. For those with mild to moderate cardiac manifestations, amoxicillin or doxycycline PO for 14 - 21 days is the preferred course of therapy and most symptoms usually resolve in 1 - 2 weeks [8]. A simple electrocardiogram proved to be an invaluable diagnostic test for this patient. Follow-up with appropriate IV and oral antibiotic therapy remains crucial [9]. The case highlights the need for prompt diagnosis and treatment of reversible cause of heart block in Lyme disease.

\section{Grant Support}

None.

\section{Conflict of Interest}

None.

\section{References}

1. Bacon RM, Kugeler KJ, Mead PS, Centers for Disease C, Prevention. Surveillance for Lyme disease-United States, 1992-2006. MMWR Surveill Summ. 2008;57(10):1-9.

2. O'Connell S, Granstrom M, Gray JS, Stanek G. Epidemiology of European Lyme borreliosis. Zentralbl Bakteriol. 1998;287(3):229-240.

3. Lindgren E, Jaenson T. Lyme borreliosis in Europe: influences of climate and climate change, epidemiology, ecology and adaptation measures. WHO/Europe Report. 2006:7-10.

4. Centers for Disease Control and Prevention. Lyme disease Data: Cases by Year 2003-2012. http://www.cdc. gov/lyme/stats/index.html?s_cid=cs_281.

5. SteereAC. Lyme disease. NEngl J Med. 2001;345(2):115125.

6. Wilske B. Epidemiology and diagnosis of Lyme borreliosis. Ann Med. 2005;37(8):568-579.

7. Steere AC, Batsford WP, Weinberg M, Alexander J, Berger HJ, Wolfson S, Malawista SE. Lyme carditis: cardiac abnormalities of Lyme disease. Ann Intern Med. 1980;93(1):8-16.

8. Krause PJ, Bockenstedt LK. Cardiology patient pages. Lyme disease and the heart. Circulation. 2013;127(7):e451-454.

9. Shapiro ED. Clinical practice. Lyme disease. N Engl J Med. 2014;370(18):1724-1731. 